\title{
Sputum-based DNA methylation biomarkers to guide lung cancer screening decisions
}

\author{
Delphine Lissa, Ana I. Robles \\ Laboratory of Human Carcinogenesis, National Cancer Institute, NIH, Bethesda, USA \\ Correspondence to: Ana I. Robles, Ph.D. Laboratory of Human Carcinogenesis, National Cancer Institute, NIH, 37 Convent Dr, Rm 3060D, Bethesda \\ 20892, USA. Email: Ana_Robles@nih.gov. \\ Provenance: This is an invited Editorial commissioned by the Section Editor Chen Chen (The Second Xiangya Hospital of Central South University, \\ Changsha, China). \\ Comment on: Leng S, Wu G, Klinge DM, et al. Gene methylation biomarkers in sputum as a classifier for lung cancer risk. Oncotarget 2017;8:63978-85.
}

Submitted Oct 07, 2017. Accepted for publication Oct 09, 2017.

doi: $10.21037 /$ jtd.2017.10.78

View this article at: http://dx.doi.org/10.21037/jtd.2017.10.78

Lung cancer is the leading cause of cancer-related deaths worldwide (1). Exposure to tobacco smoke is the main risk factor (2). Early detection and timely treatment have been shown to greatly improve lung cancer survival. In 2011, the National Lung Screening Trial (NLST) reported a 20\% reduction in lung cancer mortality when low dose computed tomography (LDCT) was used repeatedly to screen highrisk individuals (i.e., 55-74 years of age, $\geq 30$ pack-year smoking history, and if ex-smokers, had quit within the past 15 years) (3). As of 2014, annual LDTC has become the only recommended screening test for lung cancer by the U.S. Preventive Services Task Force (USPSTF) in this high-risk population (4), but several concerns have been raised. Although LDCT is highly sensitive and detects early stage tumors, it has a low specificity. In the NLST study, among the $24.2 \%$ of subjects classified as being positive, $96.4 \%$ of them were eventually diagnosed with benign nodules. This high false positive rate can cause a lot of anxiety to patients and caregivers, and lead to unnecessary exposure to additional radiation or invasive procedures (i.e., bronchoscopy and surgical lung biopsy), to ultimately confirm or rule out a cancer diagnosis. A screening program only based upon LDCT has additional limitations, including the current USPSTF eligibility criteria that may only capture a third of newly diagnosed lung cancer patients (5), and the cost of yearly LDCT screens. Almost 9 million individuals satisfy the USPSTF criteria for screening in the US (6), and the projected coverage for screening will increase total Medicare expenditure by nearly $\$ 7$ billion per month over 5 years (7). Therefore, non-invasive tests are critically needed to identify individuals at higher risk that are best suited for LDCT. Development of highly sensitive risk biomarkers used prior to LDCT would reduce the number of false positive scans later on, and therefore improve the cost-effectiveness of a LDCT screening program. Screening biomarkers should complement the ability of LDCT to rule out lung cancer among high-risk individuals, and, thus, focus resources on those more likely to benefit.

Sputum-based biomarkers are gaining a lot of attention, as sputum contains exfoliated cells from the lower respiratory tract, and can be noninvasively obtained (8). Current and former smokers produce increased bronchial secretions. Among the molecular markers that can be evaluated in sputum, DNA methylation holds great promise to identify individuals at increased risk for lung cancer (9). Lung cancer has a long latency period and goes through multiple stages before the disease manifests itself. Methylation changes have been shown to occur very early during lung carcinogenesis, not only in the neoplastic lesion but also in the normal-appearing adjacent tissue, a phenomenon referred to as field cancerization (10). Cigarette smoke induces extensive methylation changes in normal bronchial epithelia that are similar to those found in lung tumors $(11,12)$. These markers may have diagnostic utility for detection of tumor-derived methylated DNA in exfoliated airway epithelium or blood (13). An outstanding question, however, has been whether these markers can help 
stratify the large number of LDCT-eligible individuals

A recent report by Leng et al. published in Oncotarget, provides evidence that a DNA methylation signature for field cancerization measured in sputum may help prioritize the selection of smokers for LDCT screening (14). The authors compare the methylation of an 8-gene panel in sputum among two cohorts of cancer-free smokers, the Lovelace Smokers Cohort (LSC) and the PLuSS cohort (1,380 and 718 current and former smokers, respectively), and a cohort of 487 surgically resected stage I patients from the ECOG-ACRIN5595 trial. The choice of these cohorts was meant to reflect the at-risk population targeted by LDCT. Smokers from the LSC and the PLuSS cohort represented individuals who should not receive LDCT, while participants of the ECOG-ACRIN5595 trial, for whom the field of cancerization remains and is reflected in the sputum, represented the higher-risk individuals who should be recommended for LDCT. To evaluate the performance of the 8-gene methylation panel in the target screening population and therefore help rule out cancer, the initial analysis was restricted to LDCT eligible smokers $(\mathrm{n}=371$ ECOG-ACRIN 5595; n=466 LSC and $\mathrm{n}=597$ PLuSS). The methylation prevalence of all 8 genes was increased in resected lung cancer patients compared to the cancer-free smokers (odds ratios ranging from 1.6 to 8.9). The 8-gene methylation panel had a better prediction accuracy than clinical risk factors alone (including age, sex and smoking status), with area under curve (AUC) of 0.82 vs. 0.76 or 0.86 vs. 0.74, comparing ECOG-ACRIN5595 patients to LSC or PLuSS subjects, respectively. Importantly, a classifier combining the 8-gene methylation panel and clinical risk factors resulted in higher disease discrimination (AUC of 0.87 or 0.90 , respectively). Setting up the sensitivity at $95 \%$, the combined classifier increased specificity from $25 \%$ (clinical risk factor only) to $54 \%$, with positive predictive values (PPV) from $47 \%$ to $58 \%$, and negative predictive values (NPV) from $88 \%$ to $94 \%$. This is particularly important because a biomarker with strong NPV would allow the clinician to confidently rule out cancer and, thus, the need for screening (15). Finally, when assessing the performance of the 8-gene methylation panel in a broader population beyond LDCT eligible smokers (i.e., $\geq 40$ years of age, $\geq 10$ packs a year smoking history), the accuracy of the combined classifier remained similar (AUC of 0.88), as of its specificity. These results suggest that the addition of the 8-gene methylation panel to clinical risk factors could help identify high-risk smokers who would have otherwise been 'missed out' under the current
USPSTF screening criteria. The authors conclude that this gene methylation panel can improve the assessment of cancer risk among smokers and prioritize those who should receive LDCT while excluding smokers at lower risk for lung cancer.

There are several limitations to this study, particularly related to marker selection and methodology. Markers included in the panel were selected based on prior candidate gene studies, rather than a comprehensive analysis of lung cancer methylation (16). Similar studies in sputum that evaluated methylation markers derived from genomewide methylation studies, including The Cancer Genome Atlas (TCGA), resulted in overall higher AUCs $(17,18)$. Regarding methodology, positive methylation was scored in this study on the basis of detection of a visible band in a gel. Thus, extensive development of this assay would be required before its clinical evaluation can be pursued. High performance PCR technologies that can be easily implemented in the clinic have been developed in recent years to detect and quantify minute amount of methylated DNA (13).

Limitations notwithstanding, the work by Leng et al. supports efforts to incorporate molecular biomarkers derived from sputum to prioritize individuals for LDCT or for routine screening of asymptomatic individuals at high-risk for smoking-related lung cancer. The panel of methylated genes should be prospectively validated in the population targeted for LDCT screening. One more tool could be then added to the toolbox of physicians to aid decision-making and bring precision medicine to lung cancer screening (19).

\section{Acknowledgements}

The authors are supported by the Intramural Research Program of the National Cancer Institute, NIH.

\section{Footnote}

Conflicts of Interest: The authors have no conflicts of interest to declare.

\section{References}

1. Siegel RL, Miller KD, Jemal A. Cancer Statistics, 2017. CA Cancer J Clin 2017;67:7-30.

2. Hecht SS, Szabo E. Fifty years of tobacco carcinogenesis research: from mechanisms to early detection and 
prevention of lung cancer. Cancer Prev Res (Phila) 2014;7:1-8.

3. National Lung Screening Trial Research Team, Aberle DR, Adams AM, et al. Reduced lung-cancer mortality with low-dose computed tomographic screening. N Engl J Med 2011;365:395-409.

4. Moyer VA, U.S. Preventive Services Task Force. Screening for lung cancer: U.S. Preventive Services Task Force recommendation statement. Ann Intern Med 2014;160:330-8.

5. Wang Y, Midthun DE, Wampfler JA, et al. Trends in the proportion of patients with lung cancer meeting screening criteria. JAMA 2015;313:853-5.

6. Doria-Rose VP, White MC, Klabunde CN, et al. Use of lung cancer screening tests in the United States: results from the 2010 National Health Interview Survey. Cancer Epidemiol Biomarkers Prev 2012;21:1049-59.

7. Roth JA, Sullivan SD, Goulart BH, et al. Projected Clinical, Resource Use, and Fiscal Impacts of Implementing Low-Dose Computed Tomography Lung Cancer Screening in Medicare. J Oncol Pract 2015;11:267-72.

8. Kim CE, Tchou-Wong KM, Rom WN. Sputum-based molecular biomarkers for the early detection of lung cancer: limitations and promise. Cancers 2011;3:2975-89.

9. Balgkouranidou I, Liloglou T, Lianidou ES. Lung cancer epigenetics: emerging biomarkers. Biomark Med 2013;7:49-58.

10. Kadara H, Wistuba II. Field cancerization in non-small cell lung cancer: implications in disease pathogenesis. Proc Am Thorac Soc 2012;9:38-42.

Cite this article as: Lissa D, Robles AI. Sputum-based DNA methylation biomarkers to guide lung cancer screening decisions. J Thorac Dis 2017;9(11):4308-4310. doi: 10.21037/ jtd.2017.10.78
11. Zöchbauer-Müller S, Lam S, Toyooka S, et al. Aberrant methylation of multiple genes in the upper aerodigestive tract epithelium of heavy smokers. Int J Cancer 2003;107:612-6.

12. Belinsky SA, Palmisano WA, Gilliland FD, et al. Aberrant promoter methylation in bronchial epithelium and sputum from current and former smokers. Cancer Res 2002;62:2370-7.

13. Lissa D, Robles AI. Methylation analyses in liquid biopsy. Transl Lung Cancer Res 2016;5:492-504.

14. Leng S, Wu G, Klinge DM, et al. Gene methylation biomarkers in sputum as a classifier for lung cancer risk. Oncotarget 2017;8:63978-85.

15. Atwater T, Massion PP. Biomarkers of risk to develop lung cancer in the new screening era. Ann Transl Med 2016;4:158.

16. Belinsky SA, Liechty KC, Gentry FD, et al. Promoter hypermethylation of multiple genes in sputum precedes lung cancer incidence in a high-risk cohort. Cancer Res 2006;66:3338-44.

17. Hulbert A, Jusue-Torres I, Stark A, et al. Early Detection of Lung Cancer Using DNA Promoter Hypermethylation in Plasma and Sputum. Clin Cancer Res 2017;23:1998-2005.

18. Diaz-Lagares A, Mendez-Gonzalez J, Hervas D, et al. A Novel Epigenetic Signature for Early Diagnosis in Lung Cancer. Clin Cancer Res 2016;22:3361-71.

19. Robles AI, Harris CC. Integration of multiple "OMIC" biomarkers: A precision medicine strategy for lung cancer. Lung Cancer 2017;107:50-8. 